\title{
Positive Psychological Change in Head and Neck Cancer populations
}

\author{
Sam Harding
}

DHealthPsych, Pines and Steps, Southmead Hospital, North Bristol Hospital Trust, Bristol, United Kingdom

Article Info

\section{Article Notes}

Received: February 13, 2018

Accepted: March 19, 2018

\section{${ }^{*}$ Correspondence:}

Dr. Sam Harding, DHealthPsych

Pines and Steps, Southmead Hospital

North Bristol Hospital Trust

Bristol, BS10 5NB, United Kingdom; Telephone: +44 (0) 117 414 3957, E-mail: sharding.jb@gmail.com

(C) 2018 Harding S. This article is distributed under the terms of the Creative Commons Attribution 4.0 International License.

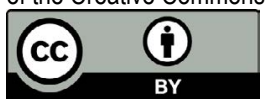

\section{Keywords}

Head Neck Cancer

Positive Psychological Change

Posttraumatic Growth

Benefit Finding

\section{ABSTRACT}

Head and neck cancer (HNC) carry a high level of morbidity and mortality, but the impact of HNC on survivors differs widely among individuals, and a significant number of them suffer from negative psychological effects of the disease. However, some people report a significant positive effect of experiencing $\mathrm{HNC}$ and its treatment.

This review looks at demographic, clinical and psychological factors associated with positive psychological change (PPC) in HNC populations.

Eight quantitative manuscripts were identified as reporting on PPC in HNC. These studies were split between recruiting participants via cancer clinics and postal surveys, and the majority use a cross-sectional study design.

Demographic factors across the papers showed similar patterns of relationships across PPC; that higher education/qualification and cohabitation/ marriage are associated with increased PPC. Limited research reported longitudinal patterns of change and showed that for people with lower stage tumours and those who only had a surgical intervention greater PPC developed over time. Multivariable modeling adjusting for psychosocial variables found that PPC had a quadratic relationship with time since diagnosis, increasing initially and leveling off after 18 months.

Further research would aid the identification of bio-psychosocial factors that influence the development of PPC and inform the development of rehabilitation interventions while enabling consideration of the natural development of the phenomenon.

\section{Introduction}

There is evidence from the literature that some people report benefit from illness ${ }^{1-6}$. In some cases, these benefits go some way to mitigating the negative consequences of illness, but there are also instances where people report an overall benefit of being ill. Positivity in adversity has been cited in the context of other stressful life events such as combat and imprisonment, divorce, care giving and bereavement ${ }^{7}$. Stress-related growth in adversity is reported to be 'remarkably common'?

There is a growing body of literature supporting the suggestion that a stressful or traumatic event may be a catalyst for positive psychological change ${ }^{8,9}$. In 1991 Yalom and Lieberman ${ }^{10}$ used the term 'positive psychological changes' to refer to positive changes in the perceptions of oneself, social relationships with family and friends and life priorities and appreciation of life. These positive changes, which have also been referred to as 'perceived benefits', 'benefit finding, 'thriving', 'stress-related growth', 'adversarial growth', 'post- 
traumatic growth', or 'existential growth', may concern changes in the perceptions of oneself, social relationships with family and friends and life priorities and appreciation of life. The term 'Post-traumatic growth' is widely used due to its ability to describe the need for individuals to have experienced trauma before they experience positive change over time. However, premininent researchers in this field, Tedeschi and Calhoun, have suggested this these terms are roughly synomous ${ }^{11}$. In this paper, positive psychological change (PPC) will be used unless reporting data directly from a journal article where they use another term such as PTG. The choice of PPC over PTG was made due to the nature of the trauma experienced by the people with and following cancer. In presenting work on PPC to people who have received a diagnosis of head and neck cancer (HNC) the author has found that the word 'growth' has significant negative meaning, as it is a word associated with a cancerous tumour. In working with this group of people, Harding et $\mathrm{al}^{12}$ suggest that the phrase positive psychological change was better received and facilitated communication.

Within the field of cancer, breast cancer (BC) has received the greatest amount of investigation into $\mathrm{PPC}^{13-18}$. There is evidence indicating that a substantial number of $\mathrm{BC}$ survivors experience such positive changes, especially in the long term ${ }^{15-17,19,20}$. Cancer survivors from tumours in a range of locations frequently report having altered priorities including more concern for others, a greater sense of purpose and a greater appreciation of themselves and their lives life ${ }^{4,21-24}$. A challenge for HNC clinicians is to understand what factors are associated with the developed of PPC. Only eight quantitative articles have been published within the field of HNC and $\mathrm{PPC}^{25-32}$. Tables 1 and 2 provide an outline of the study designs, participants, and variable.

This over view of the current literature will describe

Table 1: Study Descriptors.

\begin{tabular}{|c|c|c|c|c|c|c|c|}
\hline Study & Author(s) & Aim of the study & Study Design & Study measures & $\begin{array}{l}\text { Demographic } \\
\text { Factors }\end{array}$ & Medical Factors & $\begin{array}{c}\text { Time of } \\
\text { measurement }\end{array}$ \\
\hline 1 & $\begin{array}{l}\text { Harrington, S., } \\
\text { McGurk, M. \& } \\
\text { Llewellyn, C.D. } \\
\text { (2008) }\end{array}$ & $\begin{array}{l}\text { 1) to determine the } \\
\text { extent to which patient } \\
\text { treated for HNC expe- } \\
\text { rience positive conse- } \\
\text { quences of their illness, } 2 \text { ) } \\
\text { to identify factors associ- } \\
\text { ated with benefit finding } \\
\text { among this patient group }\end{array}$ & $\begin{array}{l}\text { Cross-sectional } \\
\text { postal survey }\end{array}$ & $\begin{array}{l}\text { Benefit finding scale } \\
\text { (BFS), Hospital Anxiety } \\
\text { and Distress Scale } \\
\text { (HADS), Life Orien- } \\
\text { tation Test - Revised } \\
\text { (LOT-R), Brief COPE }\end{array}$ & $\begin{array}{l}\text { Age, Gender, Eth- } \\
\text { nicity, Education, } \\
\text { Employment, } \\
\text { Marital status }\end{array}$ & $\begin{array}{l}\text { Type of treatment, } \\
\text { time since last } \\
\text { treatment, diagno- } \\
\text { sis of further illness } \\
\text { since treatment, } \\
\text { site, type of cancer } \\
\text { and stage of cancer }\end{array}$ & $\begin{array}{l}0-6 \text { mths }=1 \\
6-12 \text { mths }=3 \\
13-24 m \text { ths }=7 \\
25-47 m \text { ths }=20 \\
48-72 \text { mths }=19 \\
73-121 \text { mths }=26\end{array}$ \\
\hline 2 & $\begin{array}{l}\text { Llewellyn, C.D., } \\
\text { Horney, D.J., } \\
\text { McGurk, M., } \\
\text { Weinman, J., } \\
\text { Herold, J., Alt- } \\
\text { man, K. \& Smith, } \\
\text { H.E. (2011) }\end{array}$ & $\begin{array}{l}\text { 1) to determine the } \\
\text { extent to which pa- } \\
\text { tient treated for HNC } \\
\text { experience positive } \\
\text { consequences of their } \\
\text { illness, 2) to establish the } \\
\text { relationship between BF, } \\
\text { other patient-reported } \\
\text { outcomes and predictive } \\
\text { factors such as coping } \\
\text { strategy and level of } \\
\text { optimism }\end{array}$ & $\begin{array}{l}\text { Repeated mea- } \\
\text { sures prospec- } \\
\text { tive study using } \\
\text { self-completion } \\
\text { questionnaires }\end{array}$ & $\begin{array}{l}\text { Benefit finding scale } \\
\text { (BFS), Hospital Anxiety } \\
\text { and Distress Scale } \\
\text { (HADS), Life Orientation } \\
\text { Test (LOT-R), Brief COPE, } \\
\text { Medical Outcomes } \\
\text { Short Form } 12 \text { (SF-12), } \\
\text { Two-item measure } \\
\text { derived from The Euro- } \\
\text { pean Organization for } \\
\text { Research and Treatment } \\
\text { (EORTC) of Cancer Qual- } \\
\text { ity of Life Questionnaire } \\
\text { (QLQ-C30) }\end{array}$ & $\begin{array}{l}\text { Age, Gender, Eth- } \\
\text { nicity, Education, } \\
\text { Employment, } \\
\text { Marital status }\end{array}$ & $\begin{array}{l}\text { Type of treatment, } \\
\text { site and stage of } \\
\text { cancer }\end{array}$ & $\begin{array}{l}\text { T1 = Between } \\
\text { diagnosis and } \\
\text { start of treatment, } \\
\text { T2 = } 6 \text { months } \\
\text { after completion } \\
\text { of treatment }\end{array}$ \\
\hline 3 & $\begin{array}{l}\text { Ho, S.M.Y., Rajan- } \\
\text { dram, R.K, Chan, } \\
\text { N., Samman, } \\
\text { N., McGrath, C. } \\
\text { \& Zwahlen, R.A. } \\
\text { (2011) }\end{array}$ & $\begin{array}{l}\text { Investigate if PTG occurs } \\
\text { in oral cancer patients } \\
\text { and if hope and optimism } \\
\text { shows significant positive } \\
\text { correlation with PTG }\end{array}$ & $\begin{array}{l}\text { Cross-sectional } \\
\text { postal survey }\end{array}$ & $\begin{array}{l}\text { Chinese Posttraumatic } \\
\text { Growth Inventory } \\
\text { (PTGI), Hope scale } \\
\text { (HS), Life Orientation } \\
\text { Test - Revised (LOT-R) }\end{array}$ & $\begin{array}{l}\text { Age, Gender, } \\
\text { Religion, Education } \\
\text { level, income }\end{array}$ & $\begin{array}{l}\text { Time since } \\
\text { diagnosis, stage } \\
\text { of disease, and } \\
\text { treatment type }\end{array}$ & $\begin{array}{l}\text { Mean time was } \\
3.6 y r s \text { (SD 0.34) }\end{array}$ \\
\hline 4 & $\begin{array}{l}\text { Lebel, S. Cos- } \\
\text { tonguay, M., } \\
\text { Mackness, G., } \\
\text { Irish, J., Bezjak, } \\
\text { A. \& Devins, } \\
\text { GM. (2013) }\end{array}$ & $\begin{array}{l}\text { Investigate if the relation- } \\
\text { ship between stigma and } \\
\text { subjective well-being will } \\
\text { be moderated by benefit } \\
\text { finding (the negative im- } \\
\text { pact of stigma on distress } \\
\text { and subjective well-be- } \\
\text { ing will be lower when } \\
\text { people report high levels } \\
\text { of benefit finding }\end{array}$ & $\begin{array}{l}\text { Cross-sectional } \\
\text { postal survey }\end{array}$ & $\begin{array}{l}\text { Affect Balance Scale } \\
\text { (ABS), Center for Epi- } \\
\text { demiological Studies } \\
\text { Depression Scale } \\
\text { (CES-D), Explanatory } \\
\text { Model Interview } \\
\text { Catalogue (EMIC), } \\
\text { Illness Intrusiveness } \\
\text { Ratings Scale (IIRS), } \\
\text { Posttraumatic Growth } \\
\text { Inventory (PTGI), } \\
\text { Disfigurement Scale, } \\
\text { Marlowe-Crown Social } \\
\text { Desirability Scale }\end{array}$ & $\begin{array}{l}\text { Age, Gender, Mar- } \\
\text { tial status, number } \\
\text { of children, number } \\
\text { of other people } \\
\text { in the home, } \\
\text { employment status, } \\
\text { education, annual } \\
\text { household income, } \\
\text { country of birth, } \\
\text { religion, stressful } \\
\text { life events. }\end{array}$ & $\begin{array}{l}\text { Years since diagno- } \\
\text { sis, Cancer stage, } \\
\text { treatment type }\end{array}$ & $\begin{array}{l}\text { Mean time was } \\
1.37 \text { (SD 0.84) } \\
\text { years since diag- } \\
\text { nosis }\end{array}$ \\
\hline
\end{tabular}




\begin{tabular}{|c|c|c|c|c|c|c|c|}
\hline 5 & $\begin{array}{l}\text { Leong Abdullah, } \\
\text { M.F., Nik Jaafar, } \\
\text { N.R., Zakaria. H., } \\
\text { Rajandram, R.K., } \\
\text { Mahadevan } \\
\text {,R., Mohamad } \\
\text { Yunus, M.R. } \\
\text { \& Shah, S.A. } \\
\text { (2015) }\end{array}$ & $\begin{array}{l}\text { Investigate the correla- } \\
\text { tions between PTG with } \\
\text { depression and anxiety. }\end{array}$ & Cross-sectional & $\begin{array}{l}\text { Posttraumatic Growth } \\
\text { Inventory - Short Form } \\
\text { (PTGI-SF), Hospital } \\
\text { Anxiety and Depres- } \\
\text { sion Scale (HADS) }\end{array}$ & $\begin{array}{l}\text { Age, Gender, Race, } \\
\text { Monthly income, } \\
\text { educational level, } \\
\text { marital status }\end{array}$ & $\begin{array}{l}\text { Diagnosis, duration } \\
\text { of diagnosis, } \\
\text { treatment regime, } \\
\text { Cancer stage }\end{array}$ & $\begin{array}{l}\mathrm{T} 1=\text { Within } 1 \text { year } \\
\text { of diagnosis, } \mathrm{T} 2= \\
6 \text { months follow- } \\
\text { ing T1 }\end{array}$ \\
\hline 6 & $\begin{array}{l}\text { Holtmaat, K., } \\
\text { van der Spek, } \\
\text { N., Cuijper, P., } \\
\text { Leemans, C.R. } \\
\text { \& Verdonck-de } \\
\text { Leeuw, I.M. } \\
\text { (2016) }\end{array}$ & $\begin{array}{l}\text { Investigate the occur- } \\
\text { rence of PTG among HNC } \\
\text { survivors with psycho- } \\
\text { logical distress and to } \\
\text { examine the associations } \\
\text { of PTG with sociodemo- } \\
\text { graphic and clinical fac- } \\
\text { tors, nicotine and alcohol } \\
\text { use disorders, anxiety } \\
\text { and depression disorders } \\
\text { and health-related quality } \\
\text { of life }\end{array}$ & Cross-sectional & $\begin{array}{l}\text { Posttraumatic Growth } \\
\text { Inventory (PTGI), Hos- } \\
\text { pital Anxiety and De- } \\
\text { pression Scale (HADS), } \\
\text { European Organization } \\
\text { for Research and Treat- } \\
\text { ment of Cancer Quality } \\
\text { of Life Questionnaire- } \\
\text { C30 (EORTC QLQ-C30) } \\
\text { World Mental Health } \\
\text { CIDI }\end{array}$ & $\begin{array}{l}\text { Age, Gender, mar- } \\
\text { ital status, years of } \\
\text { education, employ- } \\
\text { ment status }\end{array}$ & $\begin{array}{l}\text { Tumour location, } \\
\text { Cancer stage, } \\
\text { Treatment regime, } \\
\text { Months since } \\
\text { treatment, CIDI } \\
\text { diagnosis }\end{array}$ & $\begin{array}{l}\text { Mean time was } \\
22.4 \text { (SD 25.8) } \\
\text { months since } \\
\text { diagnosis }\end{array}$ \\
\hline 7 & $\begin{array}{l}\text { Harding, S. \& } \\
\text { Moss, T.P. (2017) }\end{array}$ & $\begin{array}{l}\text { Investigate the relation- } \\
\text { ship between biomedical } \\
\text { variable, health-related } \\
\text { quality of life, social } \\
\text { factors and subjective } \\
\text { reports of PPC }\end{array}$ & $\begin{array}{l}\text { Cross-sectional } \\
\text { postal survey }\end{array}$ & $\begin{array}{l}\text { Silver Lining Question- } \\
\text { naire (SLQ), University } \\
\text { of Washington Head } \\
\text { and Neck Caner } \\
\text { Quality of Life (UoW), } \\
\text { Medical Outcomes } \\
\text { Short Form } 12 \text { (SF-12) }\end{array}$ & $\begin{array}{l}\text { Age at diagnosis, } \\
\text { Age at time of } \\
\text { completing ques- } \\
\text { tionnaire, Gender, } \\
\text { Ethnicity, Index of } \\
\text { Multiple Depriva- } \\
\text { tion, Occupation, } \\
\text { Family Status }\end{array}$ & $\begin{array}{l}\text { Cancer stage, } \\
\text { Treatment regime, } \\
\text { Months since } \\
\text { treatment, }\end{array}$ & $\begin{array}{l}\text { Mean time was } \\
6.52 \text { (SD 2.8) } \\
\text { months since } \\
\text { diagnosis }\end{array}$ \\
\hline 8 & $\begin{array}{l}\text { Harding, S. } \\
\text { (2017) }\end{array}$ & $\begin{array}{l}\text { Investigate the pattern or } \\
\text { trajectory of development } \\
\text { of PPC over a } 5 \text { year time } \\
\text { period. } \\
\text { Investigate how biologi- } \\
\text { cal, social and psychologi- } \\
\text { cal variable are associated } \\
\text { with PPC }\end{array}$ & $\begin{array}{l}5 \text { year cross-se- } \\
\text { quential }\end{array}$ & $\begin{array}{l}\text { Silver Lining Question- } \\
\text { naire (SLQ), University } \\
\text { of Washington Head } \\
\text { and Neck Caner } \\
\text { Quality of Life (UoW), } \\
\text { Medical Outcomes } \\
\text { Short Form } 12 \text { (SF-12) }\end{array}$ & $\begin{array}{l}\text { Age at diagnosis, } \\
\text { Age at time of } \\
\text { completing ques- } \\
\text { tionnaire, Gender, } \\
\text { Ethnicity, Index of } \\
\text { Multiple Depriva- } \\
\text { tion, Occupation, } \\
\text { Family Status }\end{array}$ & $\begin{array}{l}\text { Cancer stage, } \\
\text { Treatment regime, } \\
\text { Months since } \\
\text { treatment, }\end{array}$ & $\begin{array}{l}\text { Seven time cate- } \\
\text { gories are used: } \\
\text { 1) } 3-6 \text { months } \\
\text { 2) } 7-12 \text { months } \\
\text { 3) } 13-18 \text { months } \\
\text { 4) } 19-24 \text { months } \\
\text { 5) } 25-36 \text { months } \\
\text { 6) } 37-60 \text { months } \\
\text { 7) } \geq 61 \text { months }\end{array}$ \\
\hline
\end{tabular}

Table 2: Participants and Variables.

\begin{tabular}{|c|c|c|c|c|c|c|c|c|c|}
\hline Study & Author(s) & $\begin{array}{l}\text { Participants } \\
\text { (Gender, Age) }\end{array}$ & $\begin{array}{c}\text { Time of } \\
\text { measurement }\end{array}$ & $\begin{array}{c}\text { Non-respondents / } \\
\text { Dropouts }\end{array}$ & Exclusion Criteria & Cancer Site & $\begin{array}{l}\text { Cancer } \\
\text { Staging }\end{array}$ & $\begin{array}{c}\text { Cancer } \\
\text { Treatments }\end{array}$ & $\begin{array}{l}\text { Time since } \\
\text { completion } \\
\text { of treatment }\end{array}$ \\
\hline 1 & $\begin{array}{l}\text { Harrington, S., } \\
\text { McGurk, M. \& } \\
\text { Llewellyn, C.D. } \\
(2008)\end{array}$ & $\begin{array}{l}\mathrm{N}=76 \text { (55\% re- } \\
\text { sponse rate; } 37 \\
\text { Male, } 39 \text { Female; } \\
\text { Mean Age } 66.9, \\
\text { SD 12.6, Range } \\
32-97 ; 71 \text { White) }\end{array}$ & $\begin{array}{l}0-6 \text { mths }=1 \\
6-12 \text { mths }=3 \\
13-24 \text { mths }=7 \\
25-47 \text { mths }= \\
20,48-72 \text { mths } \\
=19,73- \\
121 \text { mths = } 26\end{array}$ & $\begin{array}{l}\text { Significant differ- } \\
\text { ence between gen- } \\
\text { der in responders } \\
\text { and non-respond- } \\
\text { ers (more females } \\
\text { responding) }\end{array}$ & $\begin{array}{l}\text { Under } 18 \text { years } \\
\text { of age. Having } \\
\text { palliative treatment. } \\
\text { Recurrent diagnosis, } \\
\text { metastatic disease } \\
\text { in other parts of the } \\
\text { body (excluding neck } \\
\text { nodes), a diagno- } \\
\text { sis of lymphoma, } \\
\text { mental to cognitive } \\
\text { impairments or } \\
\text { insufficient under- } \\
\text { standing of English. }\end{array}$ & Not stated & $\begin{array}{l}\text { Stage } 1-2 \\
-N=53 \\
\text { Stage } 3-4 \\
-N=23\end{array}$ & $\begin{array}{l}\text { Surgery only - N } \\
=35 \text {, } \\
\text { Radiotherapy } \\
\text { only - } \mathrm{N}=10 \text {, } \\
\text { Surgery and } \\
\text { Radiotherapy - N } \\
=30 \text {, } \\
\text { Surgery, radio- } \\
\text { therapy and } \\
\text { chemotherapy } \\
-\mathrm{N}=1\end{array}$ & $\begin{array}{l}0-6 \text { months } \\
=1, \\
6-12 \text { months } \\
=3, \\
13-24 \\
\text { months = 7, } \\
25-47 \\
\text { months = 20, } \\
48-72 \\
\text { months = 19, } \\
73-121 \\
\text { months = 26 }\end{array}$ \\
\hline 2 & $\begin{array}{l}\text { Llewellyn, } \\
\text { C.D., Horney, } \\
\text { D.J., McGurk, } \\
\text { M., Weinman, } \\
\text { J., Herold, J., } \\
\text { Altman, K. \& } \\
\text { Smith, H.E. } \\
\text { (2011) }\end{array}$ & $\begin{array}{l}\text { T1. N = } 103 \\
\text { (73 Males, } 30 \\
\text { Females; Mean } \\
\text { Age 63, SD 13.9, } \\
\text { Range 23-91; } 93 \\
\text { White). T2. N = } \\
68 \text { (Gender, Age, } \\
\text { Ethnicity data } \\
\text { provided) }\end{array}$ & $\begin{array}{l}\text { T1 = Between } \\
\text { diagnosis and } \\
\text { start of treat- } \\
\text { ment, T2 = } 6 \\
\text { months after } \\
\text { completion of } \\
\text { treatment }\end{array}$ & $\begin{array}{l}\text { There were no sig- } \\
\text { nificant differences } \\
\text { between patients } \\
\text { included and not } \\
\text { included with } \\
\text { respect to gender, } \\
\text { stage of cancer. } \\
35 \text { people did } \\
\text { not complete the } \\
\text { second time point. } \\
\text { No information is } \\
\text { given about they } \\
\text { compared at T1 }\end{array}$ & $\begin{array}{l}\text { Under } 18 \text { years } \\
\text { of age. Having } \\
\text { palliative treatment. } \\
\text { Recurrent diagnosis, } \\
\text { metastatic disease } \\
\text { in other parts of the } \\
\text { body (excluding neck } \\
\text { nodes), a diagno- } \\
\text { sis of lymphoma, } \\
\text { mental to cognitive } \\
\text { impairments or } \\
\text { insufficient under- } \\
\text { standing of English. }\end{array}$ & $\begin{array}{l}\text { Oral Cavity } \\
-\mathrm{N}=68, \\
\text { Pharynx }-\mathrm{N} \\
=8, \text { Larynx } \\
-\mathrm{N}=19, \\
\text { Other }-\mathrm{N} \\
=8\end{array}$ & $\begin{array}{l}\text { Stage } \\
1-\mathrm{N}= \\
34, \text { Stage } \\
2-\mathrm{N}= \\
25, \text { Stage } \\
3-\mathrm{N}= \\
23, \text { Stage } \\
4-\mathrm{N}=17, \\
\text { Missing } \\
\text { data - N } \\
=4\end{array}$ & $\begin{array}{l}\text { Surgery only - } \mathrm{N}= \\
36, \text { Radiotherapy } \\
\text { only - } \mathrm{N}=25, \\
\text { Chemotherapy } \\
\text { only - } \mathrm{N}=3 \text {, Sur- } \\
\text { gery and Radio- } \\
\text { therapy - } \mathrm{N}=17, \\
\text { Radiotherapy and } \\
\text { chemotherapy - } \\
\mathrm{N}=13, \text { Surgery, } \\
\text { radiotherapy and } \\
\text { chemotherapy - } \\
\mathrm{N}=9\end{array}$ & $\begin{array}{l}\text { Six months } \\
\text { at T2 }\end{array}$ \\
\hline
\end{tabular}




\begin{tabular}{|c|c|c|c|c|c|c|c|c|c|}
\hline 3 & $\begin{array}{l}\text { Ho, S.M.Y., } \\
\text { Rajandram, } \\
\text { R.K, Chan, N., } \\
\text { Samman, N., } \\
\text { McGrath, C. \& } \\
\text { Zwahlen, R.A. } \\
\text { (2011) }\end{array}$ & $\begin{array}{l}N=50 \text { (21 Male, } \\
29 \text { Female), } \\
\text { Mean Age 60, } \\
\text { (SD 13.06) }\end{array}$ & $\begin{array}{l}\text { Mean time } \\
\text { was 3.6yrs (SD } \\
0.34 \text { ) }\end{array}$ & $\begin{array}{l}\text { No information is } \\
\text { reported }\end{array}$ & $\begin{array}{l}\text { Non-native Canton- } \\
\text { ese speakers, less } \\
\text { than } 6 \text { mths post } \\
\text { treatment comple- } \\
\text { tion, recurrence }\end{array}$ & $\begin{array}{l}\text { Oral Cavity, } \\
\text { Orophar- } \\
\text { ynx, gingi- } \\
\text { val, floor } \\
\text { of mouth, } \\
\text { tongue, sali- } \\
\text { vary glands, } \\
\text { buccal mu- } \\
\text { cosa, palate. } \\
\text { Numbers } \\
\text { at each site } \\
\text { not stated. }\end{array}$ & $\begin{array}{l}\text { Stage } \\
1-2-N= \\
41, \text { Stage } \\
3-4-N= \\
5, \text { Missing } \\
\text { informa- } \\
\text { tion }-\mathrm{N} \\
=4\end{array}$ & $\begin{array}{l}\text { Surgery only }-\mathrm{N} \\
=34, \text { Surgery and } \\
\text { Radiotherapy }-\mathrm{N} \\
=16\end{array}$ & $\begin{array}{l}\text { Mean time } \\
\text { was 3.6yrs } \\
\text { (SD 0.34) }\end{array}$ \\
\hline 4 & $\begin{array}{l}\text { Lebel, S. } \\
\text { Costonguay, } \\
\text { M., Mackness, } \\
\text { G., Irish, J., } \\
\text { Bezjak, A. \& } \\
\text { Devins, GM. } \\
\text { (2013) }\end{array}$ & $\begin{array}{l}\mathrm{N}=99(48 \\
\text { Males, } 51 \mathrm{Fe}- \\
\text { male) Mean Age } \\
61.82(\mathrm{SD} .63)\end{array}$ & $\begin{array}{l}\text { Mean time } \\
\text { was 1.37yrs } \\
\text { (SD 0.84) }\end{array}$ & $\begin{array}{l}\text { No data presented } \\
\text { between respond- } \\
\text { ers and non-re- } \\
\text { sponders }\end{array}$ & $\begin{array}{l}\text { Diagnosis of second- } \\
\text { ary ore recurrent } \\
\text { HNC, under } 18 \text { years } \\
\text { of age, illiterate, still } \\
\text { to undergo surgery, } \\
\text { more than } 3 \text { years } \\
\text { post treatment }\end{array}$ & Not stated & $\begin{array}{l}60 \% \\
\text { identified } \\
\text { as local } \\
\text { disease, } \\
40 \% \\
\text { advanced } \\
\text { disease }\end{array}$ & $\begin{array}{l}\text { Surgery only }-\mathrm{N}= \\
52, \text { Surgery plus } \\
\text { other }-\mathrm{N}=47 \\
\text { Chemo }-\mathrm{N}=7 \\
\text { Radiotherapy }-\mathrm{N} \\
=45, \text { Other }-\mathrm{N} \\
=4\end{array}$ & $\begin{array}{l}\text { Mean time } \\
\text { was 1.37yrs } \\
\text { (SD 0.84) }\end{array}$ \\
\hline 5 & $\begin{array}{l}\text { Leong Ab- } \\
\text { dullah, M.F., } \\
\text { Nik Jaafar, } \\
\text { N.R., Zakaria. } \\
\text { H., Rajan- } \\
\text { dram, R.K., } \\
\text { Mahadevan } \\
\text {,R., Mohamad } \\
\text { Yunus, M.R. } \\
\text { \& Shah, S.A. } \\
\text { (2015) }\end{array}$ & $\begin{array}{l}\mathrm{N}=50(33 \\
\text { Males, } 17 \mathrm{Fe}- \\
\text { male), Mean Age } \\
49.76 \text { (SD 11.56) }\end{array}$ & $\begin{array}{l}\mathrm{T} 1=\text { Within } \\
1 \text { year of } \\
\text { diagnosis, T2 } \\
=6 \text { months } \\
\text { following } \mathrm{T} 1\end{array}$ & $\begin{array}{l}\text { No data presented } \\
\text { between respond- } \\
\text { ers and non-re- } \\
\text { sponders }\end{array}$ & $\begin{array}{l}\text { Greater than } 1 \text { year } \\
\text { post diagnosis, dis- } \\
\text { tant metastases, in a } \\
\text { relationship for less } \\
\text { than } 6 \text { months }\end{array}$ & Not stated & $\begin{array}{l}\text { Stage } \\
1-N= \\
11, \text { Stage } \\
2-N= \\
14, \text { Stage } \\
3-N=12, \\
\text { Stage } 4- \\
N=13\end{array}$ & $\begin{array}{l}\text { No treatment }-\mathrm{N}= \\
20 \text {, Surgery only }-\mathrm{N} \\
=8, \text { Radiotherapy } \\
\text { only }-\mathrm{N}=4 \text {, Che- } \\
\text { motherapy only }-\mathrm{N} \\
=3 \text {, Surgery and } \\
\text { Radiotherapy } \mathrm{N} \\
=2 \text {, Surgery and } \\
\text { chemotherapy }-\mathrm{N} \\
=1 \text {, Radiotherapy } \\
\text { and chemotherapy } \\
-\mathrm{N}=8 \text {, Surgery, } \\
\text { radiotherapy and } \\
\text { chemotherapy }-\mathrm{N} \\
=4\end{array}$ & $\begin{array}{l}\text { T1 = Within } \\
1 \text { year of } \\
\text { diagnosis, T2 } \\
=6 \text { months } \\
\text { following } \mathrm{T} 1\end{array}$ \\
\hline 6 & $\begin{array}{l}\text { Holtmaat, K., } \\
\text { van der Spek, } \\
\text { N., Cuijper, P., } \\
\text { Leemans, C.R. } \\
\text { \& Verdon- } \\
\text { ck-de Leeuw, } \\
\text { I.M. (2016) }\end{array}$ & \begin{tabular}{|l|}
$N=74(43$ \\
Males, 31 \\
Females), Mean \\
Age 61.2 (SD 8.5)
\end{tabular} & $\begin{array}{l}\text { Mean time } \\
\text { was } 22.4 \text { (SD } \\
25.8 \text { ) months } \\
\text { since diagnosis }\end{array}$ & $\begin{array}{l}\text { No difference in } \\
\text { gender or HADS } \\
\text { score, but those } \\
\text { that declined were } \\
\text { older }(\mathrm{P}<0.05)\end{array}$ & $\begin{array}{l}\text { Less than } 1 \text { month } \\
\text { post treatment, }<7 \\
\text { on the depression } \\
\text { and/or anxiety sub- } \\
\text { scale of HADS, cogni- } \\
\text { tive dysfunction, high } \\
\text { suicide risk, psychotic } \\
\text { and/or manic signs, } \\
\text { too little knowledge } \\
\text { of Dutch to complete } \\
\text { questionnaires }\end{array}$ & $\begin{array}{l}\text { Lip, oral } \\
\text { cavity, oro- } \\
\text { pharynx, } \\
\text { hypophar- } \\
\text { ynx, larynx }\end{array}$ & $\begin{array}{l}\text { Stage } 1 \text { or } \\
2-N=33, \\
\text { Stage } 3 \text { or } \\
4-N=37, \\
\text { Unknown } \\
-N=4\end{array}$ & $\begin{array}{l}\text { Surgery }-\mathrm{N}=12, \\
\text { Radiotherapy- } \mathrm{N} \\
=27, \text { Chemo- } \\
\text { therapy }-\mathrm{N}= \\
10, \text { Combination } \\
\text { surgery and other } \\
-\mathrm{N}=25\end{array}$ & $\begin{array}{l}\text { Mean time } \\
\text { was } 22.4 \\
\text { (SD 25.8) } \\
\text { months since } \\
\text { diagnosis }\end{array}$ \\
\hline 7 & $\begin{array}{l}\text { Harding, S. } \\
\text { \& Moss, T.P. } \\
\text { (2017) }\end{array}$ & $\begin{array}{l}N=52 \text { (36 Male, } \\
16 \text { Female), } \\
\text { Mean Age } 65.63 \\
\text { (SD 10.31) }\end{array}$ & $\begin{array}{l}\text { Mean time } \\
\text { was } 6.52 \text { (SD } \\
2.8) \text { months } \\
\text { since diagnosis }\end{array}$ & $\begin{array}{l}\text { No difference be- } \\
\text { tween responders } \\
\text { and non-respond- } \\
\text { ers on medical } \\
\text { or demographic } \\
\text { factors }\end{array}$ & $\begin{array}{l}<18 \text { years old, too } \\
\text { little knowledge of } \\
\text { English to complete } \\
\text { questionnaires, } \\
\text { tumour not histolog- } \\
\text { ically diagnosed as } \\
\text { squamous cell }\end{array}$ & $\begin{array}{l}\text { Mouth, lip, } \\
\text { oral cavity, } \\
\text { salivary } \\
\text { gland, } \\
\text { pharynx, } \\
\text { nasal cavi- } \\
\text { ty, sinuses }\end{array}$ & $\begin{array}{l}\text { Stage } \\
1-\mathrm{N}=10, \\
\text { Stage } 2-\mathrm{N} \\
=1, \text { Stage } \\
3-\mathrm{N}=13, \\
\text { Stage } 4-\mathrm{N} \\
=26\end{array}$ & $\begin{array}{l}\text { Surgery }-\mathrm{N}=16, \\
\text { Surgery and ra- } \\
\text { diotherapy }-\mathrm{N}= \\
17, \text { Radiotherapy } \\
\pm \text { chemotherapy } \\
-\mathrm{N}=18\end{array}$ & $\begin{array}{l}\text { Mean time } \\
\text { was } 6.52 \text { (SD } \\
2.8) \text { months } \\
\text { since diag- } \\
\text { nosis }\end{array}$ \\
\hline 8 & $\begin{array}{l}\text { Harding, S. } \\
\text { (2017) }\end{array}$ & $\begin{array}{l}\text { Seven time } \\
\text { points: } \\
\text { 1) } 3-6 \text { months - } \\
65.59 \text { (SD 11.54) } \\
\text { 2) 7-12 months - } \\
63.43 \text { (SD 8.93) } \\
\text { 3) } 13-18 \text { months- } \\
59.41 \text { (SD 9.05) } \\
\text { 4) } 19-24 \text { months- } \\
59.55 \text { (SD 12.91) } \\
\text { 5) } 25-36 \text { months- } \\
64.95 \text { (SD 15.34) } \\
\text { 6) } 37-60 \text { months- } \\
58.87 \text { (SD 10.86) } \\
\text { 7) } \geq 61 \text { months- } \\
57.64 \text { (SD 10.69) }\end{array}$ & $\begin{array}{l}\text { Seven time } \\
\text { categories are } \\
\text { used: } \\
\text { 1) } 3-6 \text { months - } \\
N=40, \\
\text { 2) } 7-12 \text { months } \\
-N=37 \text {, } \\
\text { 3) } 13-18 \text { months } \\
-N=22 \text {, } \\
\text { 4) } 19-24 \text { months } \\
-N=11 \text {, } \\
\text { 5) } 25-36 \text { months } \\
-N=20 \text {, } \\
\text { 6) } 37-60 \text { months } \\
-N=23 \text {, } \\
\text { 7) } \geq 61 \text { months }- \\
N=25\end{array}$ & $\begin{array}{l}\text { No difference be- } \\
\text { tween responders } \\
\text { and non-respond- } \\
\text { ers on medical } \\
\text { or demographic } \\
\text { factors }\end{array}$ & $\begin{array}{l}<18 \text { years old, too } \\
\text { little knowledge of } \\
\text { English to complete } \\
\text { questionnaires, } \\
\text { tumour not histo- } \\
\text { logically diagnosed } \\
\text { as squamous cell, } \\
\text { recurrence over the } \\
\text { time of data collec- } \\
\text { tion, new tumour } \\
\text { diagnosed in any } \\
\text { location }\end{array}$ & $\begin{array}{l}\text { Mouth, lip, } \\
\text { oral cavity, } \\
\text { salivary } \\
\text { gland, } \\
\text { pharynx, } \\
\text { nasal cavi- } \\
\text { ty, sinuses }\end{array}$ & $\begin{array}{l}\text { Data for } 7 \\
\text { time cat- } \\
\text { egories is } \\
\text { presented } \\
\text { in full pa- } \\
\text { per. Data } \\
\text { for 3-6 } \\
\text { months } \\
\text { was: } \\
\text { Stage } 1 \text { - N } \\
=9 \text {, Stage } \\
2-\mathrm{N}=5 \text {, } \\
\text { Stage } 3-\mathrm{N} \\
=5, \text { Stage } \\
4-\mathrm{N}=18\end{array}$ & $\begin{array}{l}\text { Data for } 7 \text { time } \\
\text { categories is } \\
\text { presented in full } \\
\text { paper. Data for } \\
3-6 \text { months was: } \\
\text { Surgery }-\mathrm{N}=16 \text {, } \\
\text { Surgery and ra- } \\
\text { diotherapy }-\mathrm{N}= \\
14, \text { Radiotherapy } \\
\pm \text { chemotherapy } \\
-\mathrm{N}=8\end{array}$ & $\begin{array}{l}\text { Seven time } \\
\text { categories } \\
\text { are used: } \\
\text { 1) } 3-6 \\
\text { months } \\
\text { 2) 7-12 } \\
\text { months } \\
\text { 3) } 13-18 \\
\text { months } \\
\text { 4) } 19-24 \\
\text { months } \\
\text { 5) } 25-36 \\
\text { months } \\
\text { 6) } 37-60 \\
\text { months } \\
\text { 7) } \geq 61 \\
\text { months }\end{array}$ \\
\hline
\end{tabular}


which variables have been found to be associated with PPC in people following HNC. The current research literature does not provide many clear associations due to the limited number of studies. Most studies are also short duration which makes it more difficult to evaluate changes over time about identified variables.

\section{What variables are associated with $\mathrm{PPC}$ in people following treatment for $\mathrm{HNC}$}

Some variables may mediate the relationship between trauma and PPC. Within studies, these variables can be categorized as demographic, clinical and psychological.

\section{Demographic factors}

Using a cross-sectional design with mixed cancer sites, Park et $a l^{33}$ found, in a mixed cancer site study, that women consistently reported higher levels of PPC than men. However, this study was of a largely young female cohort, over a comparatively short period (1 year) which makes it difficult to extrapolate to HNC survivors or other cancer sites, especially over an extended time frame.

In contrast to this, studies across cancer sites have found no relationship between gender and PPC in colorectal cancer ${ }^{34}$, hepatobiliary (having to do with liver, bile ducts, and bile) cancer ${ }^{35}$ or $\mathrm{HNC}^{25,27,28}$. Holtmaat et $a 2^{29}$ found females developed more PPC than their male cohort in an HNC population, although no reason for this is offered.

To date, no published studies have found an impact of age on PPC in HNC, though it has been found that younger participants with $\mathrm{BC}$ reported higher levels of $\mathrm{PPC}^{36,37}$. The greater number of studies undertaken with BC patients, and the larger participant numbers in those studies (due to the greater occurrence of BC in the general population), has identified age as a factor in the trajectory of change in, and final level, of PPC in $\mathrm{BC}^{15,16}$.

No clear relationship has been found between to ethnicity and PPC. Bellizzi et al ${ }^{13}$ found that AfricanAmericans treated for BC showed higher levels of PPC than Caucasians, whereas Kent et al ${ }^{18}$ found Caucasians with BC had higher PPC than African-Americans but not higher than Hispanics ${ }^{13,18}$. Studies of PPC across other traumas also found a mixed pattern. Milam ${ }^{38}$, for example, investigated AIDS/HIV and found that African-American and Hispanic participants reported higher levels of PPC than Caucasians respondents.

Educational attainment also lacks a clear relationship with PCC. A narrative systematic review by Koutrouli et $a l^{39}$ found that most studies reported that people with $\mathrm{BC}$ and lower education levels experienced higher levels of PPC. One study of HNC found higher educational level was associated with greater $\mathrm{PPC}^{32}$ and another found no association with education ${ }^{28}$.
Three studies following treatment for HNC reported a beneficial effect of marriage or stable cohabiting over single status in the reporting of $\mathrm{PPC}^{25,26,2}$. Although when assessed longitudinally Harding ${ }^{25}$ found no impact from marital status. In a study that examined the perspectives of $\mathrm{BC}$ patients and their partners, Manne et $a l^{37}$ measured marital quality and, despite concluding that partners influenced the course of PTG over time, they were not responsible for its prediction. This suggests that a stable social support system may have advantages over and above a high-quality one-to-one interaction.

Only one HNC study assessed the impact of socioeconomic status and found that those participants with high or low socio-economic status reported greater levels of PPC than those in the middle of the scale ${ }^{25}$.

\section{Clinical factors}

Eight HNC studies have investigated clinical factors of PPC $^{25-32}$ using quantitative PPC measures. Harrington, McGurk, and Llewellyn ${ }^{27}$ did not find any relationship between PPC and treatment, time since treatment, stage of cancer or diagnosis of further illness in people treated for HNC. Leong et $a l^{31}$ did not find an association with stage of the tumour with development of PPC either. This pattern was partially reinforced by the findings of, Harding ${ }^{25}$, Harding and Moss $^{26}$, Holtmaat et $a l^{29}$ and Llewellyn et $a l^{32}$.

Ho et $a l^{28}$ found that following HNC people with more advanced cancer (stages III and IV) reported a lower levels of PPC, but different treatment modalities did not significantly influence PPC. The pattern of tumour stage was supported by the work of Harding ${ }^{25}$ and Harding and Moss $^{26}$. In relation to treatment modalities, Harding ${ }^{25}$ and Harding and Moss $^{2}$ found that participants who had surgery alone reported more positive change than both those who had surgery with radiotherapy and those who were not treated surgically, but who had received radiotherapy with or without chemotherapy.

When compared to studies undertaken in $\mathrm{BC}^{15,164}$, the eight HNC studies have small sample sizes and lack clarity over the potential impact of, and mediating factors of, comorbidities on PPC trajectories ${ }^{25-32}$.

\section{Psychological factors}

Harrington, McGurk, and Llewellyn ${ }^{27}$ recruited people with HNC and found that dispositional optimism and positive reframing could account for $23 \%$ of the variance in PPC and additionally that higher levels of religious coping were correlated with greater PPC. They did not find any relationship between PPC and anxiety, or depression. Llewellyn et al. ${ }^{32}$ supported Harrington et al's ${ }^{27}$ findings related to dispositional optimism and positive reframing, and also found that increased use of emotional support and a decrease in self-blame positively affect PPC. This 
combination of factors was found to account for $39 \%$ of PPC variance. Ho et a ${ }^{28}$ also investigated people who had been diagnosed as having HNC and found that the Hope scale, the Life Orientation Test-Revised (LOT-R), and the Post Traumatic Growth Inventory (PTGI) were all positively correlated. Results of regression analyses comparing hope and optimism in relation to PPC found that they contributed to a $25 \%$ variance of PPC as measure by the PTGI. However, only 'hope' was a significant individual indicator of PPC.

Lebel et $a^{\beta 0}$ investigated the impact of stigma as a predictor of benefit finding and although they report their results as a mixed group of Lung and HN cancer, they found that when controlled for stressful life events and matched for cancer status, stigma and benefit finding predicted well-being.

Quality of Life (QoL) is an important psychological factor, and Llewellyn et $a l^{32}$ found that an increase in emotional growth was negatively related to the mental component summary (MCS) score. This indicates that higher levels of emotional growth are associated with poorer mental health-related QoL (HRQoL), but the study by Llewellyn $e t a b^{32}$ did not use a HRQoL measure specifically designed to assess HNC HRQoL factors. Harding ${ }^{25}$ used a HNC specific measure of HRQoL and SF-12 (Table 1 \& 2) and found that several subscales related to HNC and the Physical Component scale of the SF-12 were related to the development of PPC longitudinally.

Holtmaat et $\mathrm{al}^{29}$ found that lower levels of depression as measured by that sub-scale on the hospital anxiety and depression scale combined with higher levels of social functioning resulted in greater PPC.

\section{Impact of time since diagnosis or treatment completion}

A key limitation of 6 of the $8 \mathrm{HNC}$ studies is the short time frame over which data was collected ${ }^{26,28-32}$. One of those that looked at a greater time span only measured data once, so a trajectory of PPC developed could not be assessed ${ }^{27}$. To date, only Harding ${ }^{25}$ has tried to determine a longitudinal trajectory of the development of PPC and further work is needed to examine associations with trajectories of PPC over time. Harding ${ }^{25}$ goes some way to examine this, but was not able to differentiate if sub-groups with differing patterns of PPC development exist. Danhauer et al ${ }^{16}$ yielded a BC model with six PPC trajectories. They found age, race, chemotherapy status, use of adaptive coping strategies, illness intrusiveness, depressive symptoms and social support, all differed among the groups. The Danhauer et $a 1^{15,16}$ work supports the idea that there are likely to be sub-groups within the HNC population. Greater numbers of people post HNC treatment are required to more fully understand differentiating factors.

\section{Implications for clinicians}

A recent systematic review ${ }^{40}$ across cancer cohorts found that the vast majority of research has focused on $\mathrm{BC}$, and that the majority of PPC research has focused on psychologcal variables, over looking cancer-realted variables. With the small number of HNC papers it is hard to draw comparisions with other cancer cohorts, due to the different gender, ages, rates of recurrance and 5 -year surviaval times. However, the work of Danhauer ${ }^{15,16}$ and Harding ${ }^{25}$ suggest that their are similarilities in the development of PPC over time.

If PPC is going to be of benefit to health care professionals and service users, it needs to be harnessed as an intervention or elements of intervention packages. A meta-analysis assessed the relationship between intervention participation and PTG but failed to find any studies that included an outcome measure of $\mathrm{PPC}^{41}$. Roepke $^{41}$ suggests that there is a modest increase in PPC following intervention, but due to the limited research reported on the natural development and time course of PPC, it is possible that even this modest increase could be due to the passage of time. Future clinical practise needs to be mindful of these factors and include a measure of PPC in the development and delivery of interventions.

\section{Conflict of interest statement}

The author has no competing interests.

\section{Funding information}

No funding was sought to undertake this work.

\section{References}

1. Collins RL, Taylor SE, Skokan LA. A Better World or a Shattered Vision? Changes in Life Perspectives Following Victimization. Soc Cogn. 1990; 8: 263-285. doi:10.1521/soco.1990.8.3.263

2. Giovinco G, McDougald J. Logotherapy: A journey into meaning for people with AIDS. Int. Forum Logother. 1994; 17: 76-81.

3. Laerum E, Johnsen N, Smith P, et al. Myocardial infarction may induce positive changes in life-style and in the quality of life. Scand. J Prim Health Care . 1988; 6: 67-71.

4. LaFortune-Fredette SL. Breast cancer survivors: concerns and coping. Cancer Nurs. 1995; 18: 35-46.

5. O'Connor AP, Wicker CA, Germino BB. Understanding the cancer patient's search for meaning. Cancer Nurs. 1990; 13: 167-175.

6. Taylor SE. Adjustment to threatening events. A theory of cognitive adaptation. Am. Psychol. 1983; 38: 1161-1173.

7. Schaefer JA, Moos RH. Life crises and personal growth. In: Personal Coping: Theory, Research, and Application. Praeger Publishers/ Greenwood Publishing Group, Westport, CT, US, 1992; 149-170.

8. Tedeschi RG, Calhoun LG. Posttraumatic Growth: Conceptual Foundations and Empirical Evidence. Psychol Inq. 2004; 15: 1-18. doi:10.1207/s15327965pli1501_01

9. Updegraff JA, Taylor SE. From vulnerability to growth: Positive and negative effects of stressful life events. In: Loss and Trauma: General and Close Relationship Perspectives. Brunner-Routledge, Philadelphia, P.A., 2000; 3-28.

10. Yalom ID, Lieberman MA. Bereavement and heightened existential awareness. Psychiatry. 1991; 54: 334-345. 
11. Tedeschi RG, Calhoun LG. Posttraumatic Growth. In: Encyclopedia of Mental Health 2nd Edition. Editors in Chief H. Friedman. Academic Press, USA., 2015; 305-08.

12. Harding S, Sanipour F, Moss T. Existence of benefit finding and posttraumatic growth in people treated for head and neck cancer: a systematic review. PeerJ 2. 2014; e256. doi:10.7717/peerj.256

13. Bellizzi KM, Smith AW, Reeve BB, et al. Posttraumatic growth and health-related quality of life in a racially diverse cohort of breast cancer survivors. J Health Psychol. 2010; 15: 615-626. doi: $10.1177 / 1359105309356364$

14. Carver CS, Antoni MH. Finding benefit in breast cancer during the year after diagnosis predicts better adjustment 5 to 8 years after diagnosis. Health Psychol Off J Div Health Psychol Am Psychol Assoc. 2004; 23: 595-598. doi:10.1037/0278-6133.23.6.595

15. Danhauer SC, Case LD, Tedeschi R, et al. Predictors of posttraumatic growth in women with breast cancer. Psycho-oncology. 2013a; 22: 2676-2683. doi:10.1002/pon.3298

16. Danhauer SC, Russell G, Case LD, et al. Trajectories of Posttraumatic Growth and Associated Characteristics in Women with Breast Cancer. Ann Behav Med Publ Soc Behav Med. 2015; 49: 650-659. doi:10.1007/ s12160-015-9696-1

17. Danhauer SC, Russell GB, Tedeschi RG, et al. A longitudinal investigation of posttraumatic growth in adult patients undergoing treatment for acute leukemia. J Clin Psychol Med Settings. 2013b; 20: 13-24. doi:10.1007/s10880-012-9304-5

18. Kent EE, Alfano CM, Smith AW, et al. The roles of support seeking and race/ethnicity in posttraumatic growth among breast cancer survivors. J Psychosoc Oncol. 2013; 31: 393-412. doi:10.1080/0734 7332.2013.798759

19. Thornton AA. Perceiving benefits in the cancer experience. J Clin Psychol Med Settings. 2002; 9: 153-165.

20. Tomich PL, Helgeson VS, Nowak Vache EJ. Perceived growth and decline following breast cancer: a comparison to age-matched controls 5-years later. Psycho-oncology. 2005; 14: 1018-1029. doi:10.1002/pon.914

21. Dow KH, Ferrell BR, Haberman MR, Eaton L. The meaning of quality of life in cancer survivorship. Oncol Nurs Forum. 1999; 26: 519-528.

22. Ferrell BR, Grant M, Funk B, et al. Quality of life in breast cancer. Part II: Psychological and spiritual well-being. Cancer Nurs. 1998; 21: 1-9.

23. Pelusi J. The lived experience of surviving breast cancer. Oncol Nurs Forum. 1197; 24: 1343-1353

24. Schroevers MJ, Ranchor AV, Sanderman R. The role of age at the onset of cancer in relation to survivors' long-term adjustment: a controlled comparison over an eight-year period. Psycho-oncology. 2004; 13: 740-752. doi:10.1002/pon.780

25. Harding SA. The trajectory of positive psychological change in a head and neck cancer population. Int J Oral Maxillofac Surg. 2017; pii: S0901-5027(17)31634-X. doi: 10.1016/j.ijom.2017.09.010. [Epub ahead of print]

26. Harding SA, Moss T. The impact of treatment for head and neck cancer on positive psychological change within a year of completing treatment. Int J Oral Maxillofac Surg. 2018; 47(3): 302-308. doi: 10.1016/j.ijom.2017.07.023.
27. Harrington S, McGurk M, Llewellyn CD. Positive consequences of head and neck cancer: key correlates of finding benefit. J Psychosoc Oncol. 2008; 26: 43-62. doi.org/10.1080/07347330802115848

28. Ho S, Rajandram RK, Chan N, et al. The roles of hope and optimism on posttraumatic growth in oral cavity cancer patients. Oral Oncol. 2011; 47: 121-124. doi:10.1016/j.oraloncology.2010.11.015

29. Holtmaat K, van der Spek N, Cuijpers P, et al. Posttraumatic growth among head and neck cancer survivors with psychological distress. Psycho-oncology. 2017; 26(1): 96-101. doi:10.1002/pon.4106

30. Lebel S, Feldstain A, McCallum M, et al. Do behavioural self-blame and stigma predict positive health changes in survivors of lung or head and neck cancers. Psychol Health. 2013; 28(9): 1066-81. doi: 10.1080/08870446.2013.781602

31. Leong AMFI, Nik Jaafar NR, Zakaria H, et al. Posttraumatic growth, depression and anxiety in head and neck cancer patients: examining their patterns and correlations in a prospective study. Psychooncology. 2015; 24: 894-900. doi:10.1002/pon.3740

32. Llewellyn CD, Horney DJ, McGurk M, et al. Assessing the psychological predictors of benefit finding in patients with head and neck cancer. Psycho-oncology. 2013; 22: 97-105. doi:10.1002/pon.2065

33. Park CL, Edmonson D, Fenster JR, et al. Meaning Making and Psychological Adjustment Following Cancer: The mediating roles of growth, life meaning and restored just-world beliefs. J Consult Clin Psychol. 2008; 76(5): 863-875. doi: 10.1037/a0013348.

34. Salsman JM, Segerstrom SC, Brechting EH, et al. Posttraumatic growth and PTSD symptomatology among colorectal cancer survivors: a 3-month longitudinal examination of cognitive processing. Psychooncology. 2009; 18: 30-41. doi:10.1002/pon.1367

35. Steel JL, Gamblin TC, Carr BI. Measuring post-traumatic growth in people diagnosed with hepatobiliary cancer: directions for future research. Oncol Nurs Forum. 2008; 35: 643-650. doi:10.1188/08. ONF.643-650

36. Low CA, Stanton AL, Thompson N, et al. Contextual life stress and coping strategies as predictors of adjustment to breast cancer survivorship. Ann Behav Med. 2006; 32: 235-244. doi:10.1207/ s15324796abm3203_10

37. Manne S, Ostroff J, Winkel G, et al. Posttraumatic Growth After Breast Cancer: Patient, Partner, and Couple Perspectives. Psychosom Med. 2004 66: 442-454.

38. Milam JE. Posttraumatic Growth Among HIV/AIDS Patients1. J Appl Soc Psychol. 2004; 34: 2353-2376. doi:10.1111/j.1559-1816.2004. tb01981.x

39. Koutrouli N, Anagnostopoulos F, Potamianos G. Posttraumatic Stress Disorder and Posttraumatic Growth in Breast Cancer Patients: A Systematic Review. Women Health. 2012; 52: 503-516. doi:10.1080 /03630242.2012.679337

40. Casellas-Grau A. Ocha C Ruini C. Psychological and clinical correlates of posttraumatic growth in cancer: A systematic and critical review. Psychooncology. 2017; 26(12): 2007-18. doi: 10.1002/pon.4426

41. Roepke AM. Psychosocial Interventions and Posttraumatic Growth: A Meta-Analysis. Journal of Consulting and Clinical Psychology. 2015; 83(1): 129-142. doi: $10.1037 / \mathrm{a} 0036872$ 